for finding something' vorkommt. S. 17, 243, 251, 252 wird gesagt, lapp. vero, viero, vierro, viro fehle in den Wörterbüchern. Es ist jedoch dasselbe Wort wie lulelappisch eärró 'Opfer; Steuer', norweg.-lapp. carro 'tax etc.' < finn. vero. (Beachte auch norweg.-lapp. vierro 'usage, usual practice; custom, habit' < finn. viero.) Das S. 18, 437 erwähnte lapp. tjelilu, tsikku(ve), sikku, tsiekku, tsieggiu bedeutet 'Opfer. spezicll Rentieropfer'. Die Vielart der Formen des Wortes rührt wohl von der "laienhaften" verschiedenen Schreibweise her; es handelt sich offensichtlich um südlapp. (E. Lagercrantz) tseìhíncE

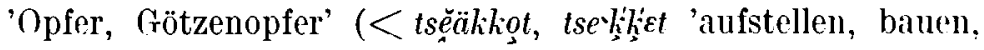
instiften' = norweg.-lapp. cag'got, ceg'git; vgl. cēggišs 'cairn to show the way on a bare mountain'). S. 100 finn. uhriailki 'Opferkiefer mit hohlem Stamm' muss heissen 'grosse Opferkiefer'. S. 99 lapp. Ruossatseärru 'rotes Herbstfjell'; norweg.lapp. ruos'sa ist '(x-shaped) cross', čcerro 'rather flot, usually wide tract high up in the mountains etc.' $S$. 252 wird aus der Lycksele-Lappmark ein (rero)gerlie (Opferstein) mit dem Namen guivie erwähnt. Es ist ein eigenartiges Zusammentreffen, dass es in Utsjoki im finnischen Lappland einen seite-Stein mit dem Namen Ivvar-kuivi, auf einem von den drei Ailigas ('Heilig')Fjellen, gibt und auch in der Mitte der Kola-IIalbinsel eine Felswand mit dem Namen Kuiv-pä $\chi$, wo der Seite den Namen Kuiv, der Legende nach 'Versteinerter Mann', trägt; vgl. auch den in den Jahren 1553-1640 bei den Lappen in der Finnmark und in Finnland gebräuchlichen Mänmernamen Kuivia (Qwiffuia, Quiffio, Quijfvi, Kniffue, Kuiuia, Kuive usw. Virittäjä 1942 , S. 65).

T. I. ITKONEN

Haxs Findeisen, Schamanentum dargestellt am Beispiel der Besessenheitspriester nordeurasiatischer Völker. Stuttgart 1957. 241 S., 14 Abb., 1 Karte.

Die Literatur, die den Schamanismus behandelt, ist recht umfangreich und in erster Linie auf Russisch geschrieben. Die erste Gesamtdarstellung davon ist in dem Werk J. G. Georgis "Beschreibung aller Nationen des Russischen Reichs" vom Jahre 1780 enthalten. Die bedeutendsten Erforscher des Schimanismus sind gewesen Bogoras, Eliade, Harra, Michailovskij, Nioradze, Ohlmarks und Šmkevič. Dr. Findeisen hat sich persönlich mit der Gedankenwelt und Tätigkeit der Schamanen unter den Jenisseiern bekanntmachen können; er hat sich, man kann sagen, praktisch gesehen mit allem, was über den 
Schamanismus geschrieben ist, vertraut gemacht und stellt darin eine Glaubensform fest, "die in Nordasien alle anderen religiösen Bereiche, Anschauungen und Sitten weitgehend durchsetzt und sich angeglichen hat." Es ist erstaunlich, dass die kleinen Völker in engen Verhältnissen und in einer rauhen Natur ein fast unvorstellbar reiches Geistesleben entwickelt haben, dessen Äusserungen naturphilosophische Mythen, Riten, Gedichte und Lieder sind.

Dr. Findeisens Werk gliedert sich in 17 Kapitel, in denen er eingehend z.B. das Werden zum Schamanen, die "Schamanenkrankheit», die Kleidung und Ausrüstung des Schamanen, die Zauhertrommel, die Beerdigung des Schamanen, die Beziehungen des verstorbenen Schamanen zu den Sippen- und Stammesgenossen, die verschiedenen Arten von Schamanen und auch das Schamanentum im Vergleich zum Mediumismus und Spiritismus behandelt.

Der Verfasser leitet die Anfänge des Schamanismus aus dem wenigstens 10 Jahrtausende zurückliegenden Jungpaläolithikum her, das seinerzeit von Spanien und Frankreich im Westen bis zum Oberlauf des Lena im Osten reichte. Auf Grund einiger Höhlenmalereien und Felszeichnungen konstatiert er mit Berufung auf die archäologischen Forschungen, dass schon in jenen fernen Zeiten die Menschen Jagdmagie trieben. Der bei den Völkern Nordeurasiens so allgemeine Bärenkult ist auch uralt. Die den Tieren, insbesondere dem Bären anheimgegebene Geisterwelt habe zur "Tierschicht" der Glaubensvorstellungen gehört, die älter zu sein scheint als der Schamanismus im exakten Sinne. Es ist eigenartig, dass den Schamanen Jagdzauber und Tierzeremoniell, Tiermythos und totemistischgenealogische Überlieferung fehlt, obgleich "das Schamanentum, d.h. die von Geistern besetzten Priestergestalten, mancherlei Elemente der Tierschicht in sich aufgenommen hat. -- _. Der Schamane ist also ein zum Besessenheitspriester gewordener jungpaläolitischer Magier.» Bei den Schamanen gibt es eine "Tiermutter" wie den mit eisernen Flügeln dargestellten adlerartigen Vogel, ferner den Elch, das Rentier, den Bären und bei den Jakuten sogar das Rind. Die Schamanen können als Tiere gegen die als Tiere vorgestellten Krankheitsgeister kämpfen und auch ihre Hilfsgeister treten oft in Tiergestalt auf, z.B. als blutdürstige Hunde. Und schliesslich stellt man sich auch die Schamanenkleidung als Tierkleid vor. - Anlässlich des oben Dargestellten könnte man freilich die Frage stellen, ob der Verfasser nicht übertreibt, wenn er den Schamanismus als etwas von der "Tierschicht" Getrenntes betrachtet. Er übergeht hier ganz und gar den lappischen noai'de, für den gerade 
charakteristisch ist, dass er, wie man sich vorstellte, im Ekstasezustand als Tier, als ein vierfüssiges (besonders als Rentierbulle), als Vogel oder Fisch wanderte. Zu den Pflichten des noai'de gehörte es auch, mit Hilfe der Zaubertrommel die Aufenthaltsorte von Wild rentierherden und anderem wichtigen Wild für die von der Dorfgemeinschaft veranstalteten Jagden in Erfahrung zu bringen. Oder sollte man die Sache so rerstehen, dass der noai' de eine primitivere Stufe des Schamanen vertritt?

Der Schamane hält sich für einen Vermittler der Creister, besonders der Ahnengeister. Wenn die Geister irgendeine Person zum Schamanenkandidaten erwählen, kann er sich dessen nicht weigern, ohne eine Strafe zu erleiden, die ihn selbst, aber auch seine Angehörigen, wie man erzählt, sogar den ganzen Stamm trifft. Schamane kann man auf verschiedene Weise werden. Im allgemeinen kommen hierbei verschiedenartige, als Personen gedachte Geisterwesen in Frage, durch die der Kandidat die notwendigen Eigenschaften erlangt. Solche Wesen sind u.a. die Ahnengeister, im Zusammenhang mit der Sippenorganisation; der Geist irgendeines verstorbenen Schamanen; ein Geist der Oberen Welt (des Hirnmels), der von dem Kandidaten Besitz ergreift; Besitzergeister eines Berges oder Gewässers, die sich in einem neuen Schamanen manifestieren wollen, und auch die "Tiermutter", die die Seele des Kandidaten in die Untere Welt führt und sie dort nährt. Später entstanden scheint die Weise, dass der Kandidat bei einem amtierenden Schamanen lernt.

Die sogen. Schamanenkrankheit, die als kennzeichnend für den, der sich der Tätigkeit eines Zauberers widmen will, betrachtet wird, ist auf verschiedene Weise erklärt worden. Am allgemeinsten ist die Auffassung, dass sie eine Art Epilepsie ist oder auch "arktische Hysterie", ja sogar eine Art Geisteskrankheit. Dr. Findeisen meint, dass Uno Harva der Wahrheit nahe gekommen sei durch seinen Ausspruch: "Wenn auch die krankhaften Erscheinungen die Voraussetzung für den Beruf des Schamanen sind, so ist es doch klar, dass man die sibirischen Schamanen, die sich eines grossen Ansehens erfreuen, nicht bloss für Geisteskranke halten darf." Die Schamanen haben Halluzinationen, Unterhaltungen mit Geistern und Ohnmachtszustände. Ein eigentümlicher Zug an dem werdenden Schamanen ist das mystische, vorgestellte Zerstückeltwerden, das u.a. bei den Tungusen, Burjaten, Jakuten und einigen altaischen Völkern vorkommt. Die "Tiermütter" und die Ahnengeister zerstückeln den Leib des auf das Schamanentum sich Vorbereitenden, ja sie können sogar auch sein Fleisch und 
Blut geniessen, damit er Krankheiten und 'Tod in jeder Ltinsicht kennen lerne. Schliesslich fügen die Geister die Knochen wieder in der richtigen Ordnung zusammen, lassen Fleisch um sie herumwachsen und das Leben in sie zurückkehren. Diese I.eidensprozedur dauert einige Tage hindurch und der "Patient" liegt dahei wie tot, ja er kinn sogar stigmatisiert werden.

Bei einigen sibirischen Völkern konmt eine sehr komplizierte Zeremonie vor, bei der ein berühmter Schamane den Kandidaten für seine Tätigkeit weiht. Mehr entwickelt findet man sie bei den Burjaten, und der Verfasser heisst die Vermutung Harvas gut, dass in diesen Weihezeremonien Einflüsse des I uamaismus (Weihen zum Vollmönch), vielleicht sogar ganz ferne Ausstrahlungen der Mithrasmysterien vorhanden sind.

Die nordasiatische Schamanenkleidung symbolisiert verschiedene 'Tiere. Vom Gebiet der Samojeden bis zur Mandschurei figuriert als solches ein hirschartiges 'Tier: bei den Samojeden, Jenisseiern und Tungusen das Rentier; bei den Osttungusen und Burjaten der Rehbock; bei den Jakuten, Dolganen und den altai-sajanischen Stämmen der Vogel (u.a. Uhu und Weiher); bei den Mandschu, Solonen und transbaikalischen Tungusøn wiederum beide Tiergattungen. Der Verfasser hält die Vogelsymbole für älter als die der Vierfüssler, weil der Schamane inf seiner Reise in den Himmel oder in die Unterwelt einen geflügelten Begleiter mit grösserem Nutzen gebranchen konnte. Der Vogel ist zuweilen eine 'Tiermutter, und in dieser Eigenschaft kann auch ein geflügeltes Rentier auftreten.

Harva führte den Ursprung der Schamanenkleidung auf die Maske, die das Gesicht oder den Kopf bedeckte, zurück. Ihr Zweck war, die Geister zu verscheuchen. Dr. Findeisen macht darauf aufmerksam, dass auf den paläolithischen Bildern tanzende Figuren dargestellt sind, bei denen der ganze Oberkörper mit einem Fell bekleidet und der Kopf mit Hörnerm versehen ist wie bei einer Gemse; es kommt auch ein Mann mit einer Hirschdecke vor, die in einen Pferdeschweif mündet und auch die Hände sind mit Fell bedeckt; weiterhin findet man da einen tanzenden Mann, der als Wildschwein gekleidet ist, und einen anderen, der in ein Bisonfell gehüllt ist. Als Alter eines dieser Bilder lässt sich ungefähr das Jahr $8500 \mathrm{v}$. Chr. bestimmen, so dass man das Altrr der nordasiatischen Schamanenkleidung auf über 10000 Jahre schätzen könme. - Fs scheint jedoch einigermassen gewagt, heut herrschende Bräuche, wie der Verfasser es tut, ohne weiteres auf so ferne Zeiten zurückzuführen, weil uns über die Zwischenzeit entsprechende Nachrichten fehlen. Es kommt noch der Umstand hinzu, dass 
der der "Tierschicht" nahe stehende lappische Zauberer nur dieselbe Kleidung gebrauchte wie seine übrigen Stammesgenossen. Man muss wohl einstweilen die Frage, ob die Kopfbedeckung älter sei als die übrige Kleidung des Schamanen, für ungelöst halten.

An der Schamanenkleidung befinden sich aus Eisen geschmiedete Imitationen von Teilen des tierischen oder menschlichen Knochengerüstes. Bei den Jenisseiern bedeuten diese "Knochen» das Knochengerüst des Schamanen selbst, denn als Körperwesen könnte er die Reise in die Oberwelt nicht durchführen, aber das eiserne Knochengerüst macht es ihm möglich. In den verschiedenen Gegenden wird die Bedeutung der "Knochen" auf verschiedene Weise erklärt. Im übrigen kann man als die Zeit, in der man im südlichen Sibirien anfing, Eisen zu schmieden, vielleicht schon das 5. prähistorische Jahrhundert bestimmen (nach Rudenko). Der Schmied stand in hohen Ehren. Ein jakutisches Sprichwort lautet: Schmiede und Schamanen stammen aus dem gleichen Nest. Die Burjaten am Baikal schätzen Schmiede und Schamanen gleich hoch und nennen die einen die "weissen" und die anderen die "schwarzen". Diese Schamanen zweierlei Art haben auch die .Jakuten und die Altai-Tataren.

Wenn der Schamane gestorben ist, wird er mit feierlicheren Zeremonien als andere bestattet, und vielerorts glaubt man an seine Wiedergeburt ebenso wie daran, dass er weiterhin für seine Sippen- und Stammesgenossen Sorge trägt. Im Himmel, (bzw. oben oder dort, wo die Grenze zwischen Tag und Nacht liegt) steht ein grosser Schamanenbaum, und in seinen Zweigen sind Nester, in denen die Seelen der Schamanen grossgezogen werden, weiter oben die der grossen, weiter unten die der kleineren; diese ernährt die Tiermutter. Die Idee von ihr geht vielleicht auf totemistische Vorstellungen zurück, während der Gedanke des Schamanenbaums möglicherweise von dem Weltbaum der vorderasiatischen Hochkulturen stammt.

Von den Schamanen gibt es verschiedene Stufen. So haben die Golden des Amurgebietes von ihnen drei verschiedene Arten: 1) solche, die nur Krankheiten heilen, 2) solche, die die Gedenkfeiern für die Verstorbenen einige Tage nach deren Tode veranstalten, 3) solche, die die Seele des Verstorbenen in das Jenseits begleiten. Die zuletztgenannte Klasse ist natürlich die am höchsten geschätzte. - Man kann sagen, dass ein richtiger Schamane das Faktotum seines Stammes ist: er ist Seelsorger, Arzt, Opferpriester, Hymnendichter, Sänger, Komponist, Tänzer, Kenner und Sachwalter der Stammestraditionen, ja oft sogar der politische Führer seiner Sippe oder seines Stammes.

Von der Zaubertrommel hat Harva angenommen, dass sie 
ältel als die Schanmanenkleidung sei. Findeisen trägt die gegenteilige Ansicht vor, indem or davon ausgeht, dass auf den paläolithischen Bildern keine Trommeln zu sehen sind, wohl aber die Kleidung. Er gibt allerdings zu, dass die Lappen in dieser Beziehung eine gewisse Schwierigkeit bereiten, da sie zwar die, Trommel, aber keine Zaubererkleidung hatten. Als Stütze seiner Theorie bringt der Verfasser vor, dass unter den in 5. vorchristlichen Jahrhundert in Pazyryk im Altai gemachten reichlichen Funden keine Trommeln vorkommen (der Verfasser sagt nicht, ob Schamanenkleidung oder ihre Teile gefunden wurden). Die nordasiatischen Trommeln sind Rahmentrommeln, oval bei den Tungusen, Jakuten, den Völkern am Amur und den Jukagiren, rund bei den Ostjaken, Wogulen, Samojeden, Jenisseiern und Altaiern. Die Trommel ist ein "beseelter" Gegenstand. Z.B. bei den Jakuten betrachtet man sie als ein Tier, auf dem der Schamane in die Geisterwelt gelangt, und dieselbe Auffassungsweise kommt auch in den Legenden der Burjaten zum Vorschein. Im Alten Orient kann man das Vorkommen der Rahmentrommel schon ungefähr 2 Jahrtausende vor Chr. feststellen. Dr. Findeisen scheint jedoch zu der Annahme zu neigen, dass sie einem alten Einfluss aus dem chinesischen Raum zuzuschreiben ist. China ist ja das 》Gelobte Land» der Trommeln, wo man an Pauken und Trommeln ïber 40 verschiedene Arten kennt! - Eire Schwierigkeit bereiten jedoch wieder die Lappen, deren Zaubertrommel kaum aus China hergeleitet werden kann. Ihre Trommel ist oval, entweder eine Rahmentrommel oder aus einem Baumstumpf ausgehöhlt, schalenförmig. Wie auch der Verfasser erwähnt, hat man auf der an der Küste ron Murmansk liegenden Insel Olenij-Ostrov bei Funden aus Gräbern der Lappen (?) ungefähr aus dem Jahr $500 \mathrm{v}$. Chr. einen T-förmigen Gegenstand aus Rentiergeweih bergen können, der dem späteren lappischen Trommelschlägel sehr ähnlich ist. Der Ursprung der nordeurasischen Trommel dürfte dunkel bleiben, besonders da man weiss, dass auch die Eskimos Amerikas einen entsprechenden Gegenstand besitzen.

Âm Ende seines Buches unterzieht der Verfasser die Beobachtungen, die über den Charakter und das Seelenleben der Schamanen gemacht worden sind, einer eingehenden Prüfung und kommt zu dem Schluss, dass die Schamanen im allgemeinen keine Epileptiker, Hysteriker oder Psychopaten im modernen psychiatrischen Sinne sind, weil sie imstande sind, neben ihrem "Amt" auch dem gewöhnlichen Lebenserwerb durch Jagd, Fischerei und Viehzucht nachzugehen in derselben Weise wie die gewöhnlichen Leute des Volkes. Dr. Findeisen vergleicht 
sie, wie es scheint mit vollem Recht, mit den spiritistischen Medien unserer Tage. Er hat sich mit der psychiatrischen Literatur vertraut gemacht und ausserdem in den Jahren 1949 - 52 protokollierte Sitzungen mit einem deutschen Medium abgehalten, um sich mit wissenschaftlicher Genauigkeit mit dem Spiritismus bekanntzumachen. Die Ergebnisse scheinen anch seine Diagnose über die Schamanen zu bestätigen.

Es ist dem Verfasser wirklich geglückt, tief in das Wesen des Schamanismiss einzudringen. Einen guten Hintergrund für seine Schlussfolgerungen geben seine recht ausführlichen Schilderungen und Analysen der Tätigkeit und der Erlebnisse zahlreicher mit Namen genannter Schamanen, die solche auf ihren Reisen in den Himmel und die Unterwelt hatten, vor allem auf Grund der bei verschiedenen Völkern gesammelten Erzählungen, Lieder und Legenden. Er hat von seinem Thema eine umfangreiche Zusammenfassung geboten und eine Menge auch ganz neue Gesichtspunkte vorgebracht, die hoffentlich Anregung zur Weiterführung der Diskussion anf diesem interessanten Forschungsgebiet geben.

\section{T. I. ITKONEN}

Ivar Paulson, Die primitiven Seelenvorstellungen der nordeurasischen Völker. Eine religionsethnographische und religionsphänomenologische Untersuchung. The Ethnographical Museum of Sweden, Stockholm (Statens Etnografiska Museum),Monograph Series - Publication No. 5. Stockholm 1958. 407 S. (Diss.)

Mit dieser geographisch weitreichenden und inhaltsreichen Arbeit tritt Ivar Paulson in die Reihe der Gelehrten, die ihr Interesse der ethnologischen und religionsgeschichtlichen Erforschung der nördlichen Völker von Europa und Asien (Sibirien, teils auch Zentral-Asien) widmen. In diesem gewaltig grossen Gebiet, das sich in westöstlicher Richtung vom Atlantischen Ozean bis zum Stillen Ozean und in nordsüdlicher Richtung vom Eismeer bis zu den nordmongolischen Steppen erstreckt, handelt es sich um sechs grosse Völkerfamilien (die finnisch-ugrische, samojedische, tungusische, türkische, mongolische, sowie die sog. paläosibirische), die in ihren Sprachen und Sitten recht stark voneinander abweichen. Abgesehen davon gibt es aber doch Kulturelemente und Vorstellungen, die mit ihrer Gleichförmigkeit bei allen diesen Völkern überraschen. Die vorliegende Arbeit ist nicht die erste, die sich das Ziel setzt, die religiösen Vorstellungen der nordeurasischen Völker zu untersuchen. Als 